\title{
Gold Complexes and Activated Carbon
}

\author{
A LITERATURE REVIEW
}

\section{Gloria J. McDougall}

Klipfontein Organic Products, Kempton Park, South Africa

and Robert D. Hancock

Sentrachem Research Group, Chemistry Department, University of the Witwatersrand, Johannesburg, South Africa

Exciting new developments are taking place in the extractive metallurgy of gold, which are based upon the adsorption of the metal or its complexes on carbon and subsequent elution. Despite the efforts of investigators over a period of almost 70 years, however, the mechanisms of the adsorption and elution processes have not yet been established unequivocally. The literature relating to them is reviewed here.

Metallurgical interest in activated carbon, notably in Australia, can be traced back as far as, 1880 when Davis (1) patented a process in which wood charcoal was used for the recovery of gold from chlorination leach liquors. Soon after the discovery by MacArthur and the Forrest brothers in 1890 that cyanide was a good solvent for gold (2), Johnson in 1894, patented the use of wood charcoal for the recovery of gold from cyanide solutions (3). Cyanidation gradually replaced chlorination and, although charcoal was never extensively used for the recovery of gold from cyanide leach liquors, the process, due largely to the efforts of Moore and Edmands (4), reached its highest state of efficiency at the Yuanmi Mine, Australia, in 1917. In this process, the pregnant solution was pumped through three successive filters that contained fine wood charcoal. Since the charcoal was prepared simply by heating wood to red heat, quenching it in water and then grinding the charcoal fine, this product could not have developed the high surface area and porosity typical of modern activated carbons, and therefore possessed a very low gold capacity. Another serious disadvantage of this earlier process was the lack of a suitable procedure for elution or desorption of the gold values from the loaded charcoal, so that the gold had to be recovered by smelting after burning the charcoal to ashes. Very low loadings were achieved and a large amount of ash was obtained that contained only about 10 to 20 per cent gold. Since these problems remained unsolved and the technology of zinc cementation advanced during the early years of the cyanidation process, interest in carbon for gold recovery declined rapidly.

In recent years, many processes (5) have been proposed for the recovery of gold from cyanide solutions and pulps in which granular or powdered activated carbon is used as an adsorbent for the gold. Undoubtedly, that which enjoys the greatest prominence at present is the carbon-in-pulp (CIP) process. In this, carbon granules are added directly to the cyanided pulp and moved counter-current to it. The goldloaded carbon is later recovered by screening. Much of the technology on which the CIP process is based was developed by Zadra et al. $(6,7)$ in the laboratories of the United States Bureau of Mines, and it was this technology that paved the way for the installation in 1973 of the first large-scale CIP plant by the Homestake Mining Company in the U.S.A. (8). The process, which has four adsorption stages, incorporates a procedure for the simultaneous elution and electrowinning of the gold, and the carbon is returned to the adsorption circuit after suitable re-activation. A simplified flow-sheet for the Homestake adsorption circuit is shown in Figure 1. This plant has operated successfully from its inception and has effectively demonstrated the viability of the CIP process at commercial level.

In recent years, much interest in the CIP process has been generated in South Africa. Excellent results obtained on a pilot-plant scale (9) at various gold mines on the Witwatersrand, for example Durban Roodepoort Deep and Grootvlei, and also at the Fairview Mine in the Barberton area, are responsible for the fact that at least five South African gold mines are planning to install large-scale fully integrated CIP plants by the end of 1981, and a further five are committed to do so by the end of 1982 . Two main factors are responsible for the rapid development of CIP and related processes in recent years. In the first place, carbon products that combine high gold activity with 


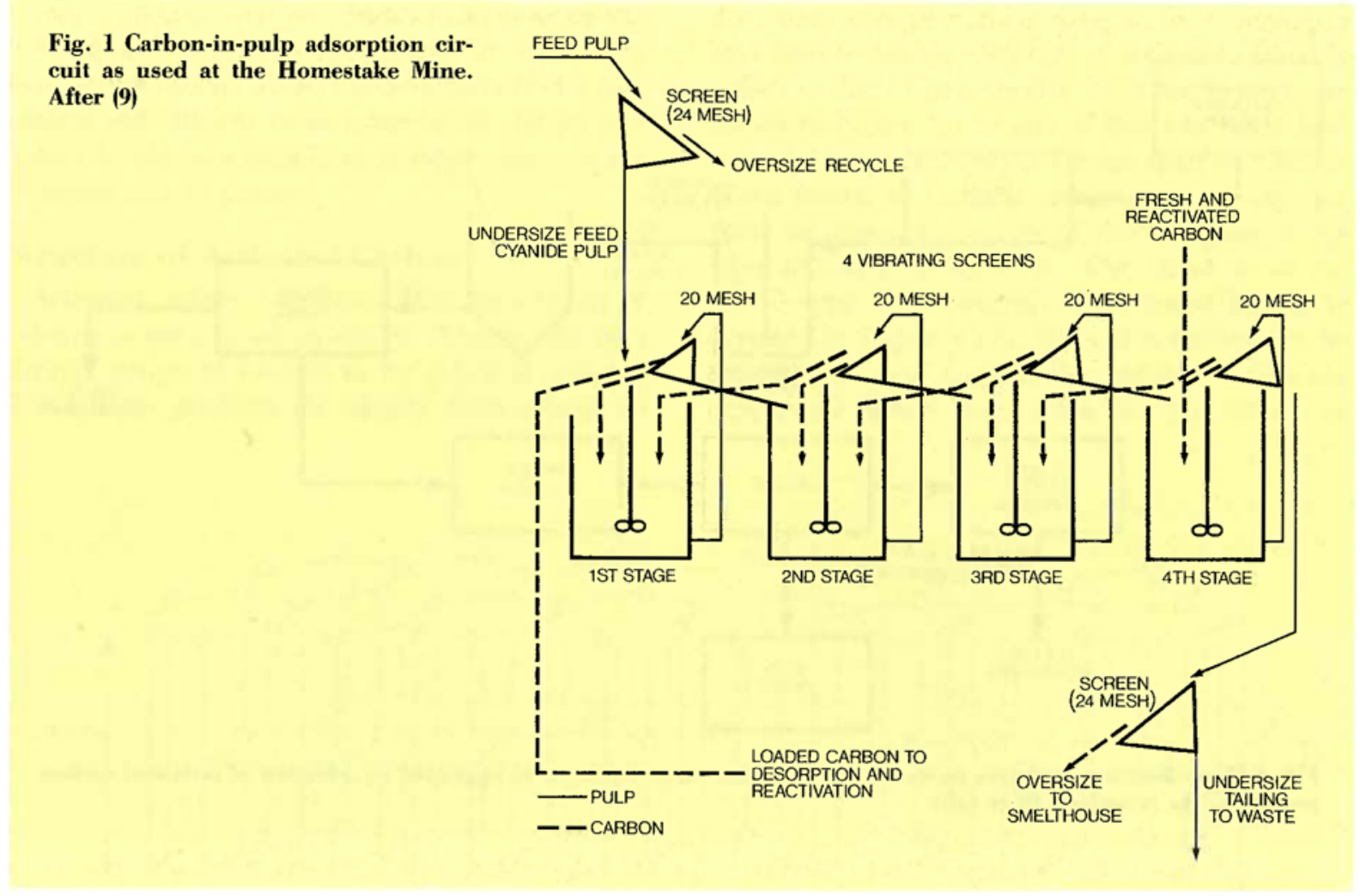

the superior abrasion resistance necessary in order to minimize the loss of gold that would result if softer carbons were employed, became available in large quantities only in the early sixties. Excellent such products can be manufactured from coconut shells and coal. Secondly, efficient elution procedures which allow the carbon to be re-used after suitable reactivation, were developed only in comparatively recent times. The gold cyanide is tenaciously held by the carbon and, in the currently employed elution procedures, the gold values are desorbed with hot (about $90^{\circ} \mathrm{C}$ ) water after pretreatment of the carbon with a solution of sodium hydroxide and sodium cyanide $(5,7,10)$ or simply by circulating a hot caustic cyanide solution ( 1 per cent $\mathrm{NaOH}$ and 0.2 per cent $\mathrm{NaCN}$ ) through a column of gold-loaded carbon (7). The gold is recovered from the eluant by electrolysis. The rate at which the gold is desorbed from the carbon can be substantially improved by modification of the water or caustic cyanide eluant, by the addition to it of 5 to 20 per cent methanol or ethanol for example (11), or by conducting the elution under pressure at temperatures between 100 and $180^{\circ} \mathrm{C}(10,12)$.

The CIP process has several important advantages over the conventional zinc cementation route $(5,9)$. These are:

(1) The ability of carbon to adsorb gold (and silver) cyanide is not affected by any of the common constituents of cyanide leach liquors, some of which (for example, the cyanide complexes of copper and nickel) are detrimental to the recovery of gold by zinc precipitation

(2) The carbon granules are added directly to the cyanided pulp, which obviates the need for the expensive filtration and clarification stages that are required in the cementation process

(3) The soluble gold losses are usually significantly lower than on a conventional plant.

Therefore, it is generally believed that CIP offers economic advantages over zinc precipitation, not only with respect to higher gold recoveries, but also in terms of lower capital and operating costs.

As far as current and future developments are concerned, a number of interesting processes warrant mention.

First, it has been demonstrated that activated carbon is an excellent scavenger for small concentrations of dissolved gold $(0.2 \mathrm{mg} / 1$ or less) $(13,14)$, and therefore it is likely that activated carbon will find increased use in finishing applications, such as in the removal of dissolved gold cyanide from gold plant effluents and dam return water. It is convenient to conduct such operations using the column technique, with either a fixed or a fluidized bed of granular carbon, the choice of loading technique depending on the amount of solids present in the gold-containing solution. 


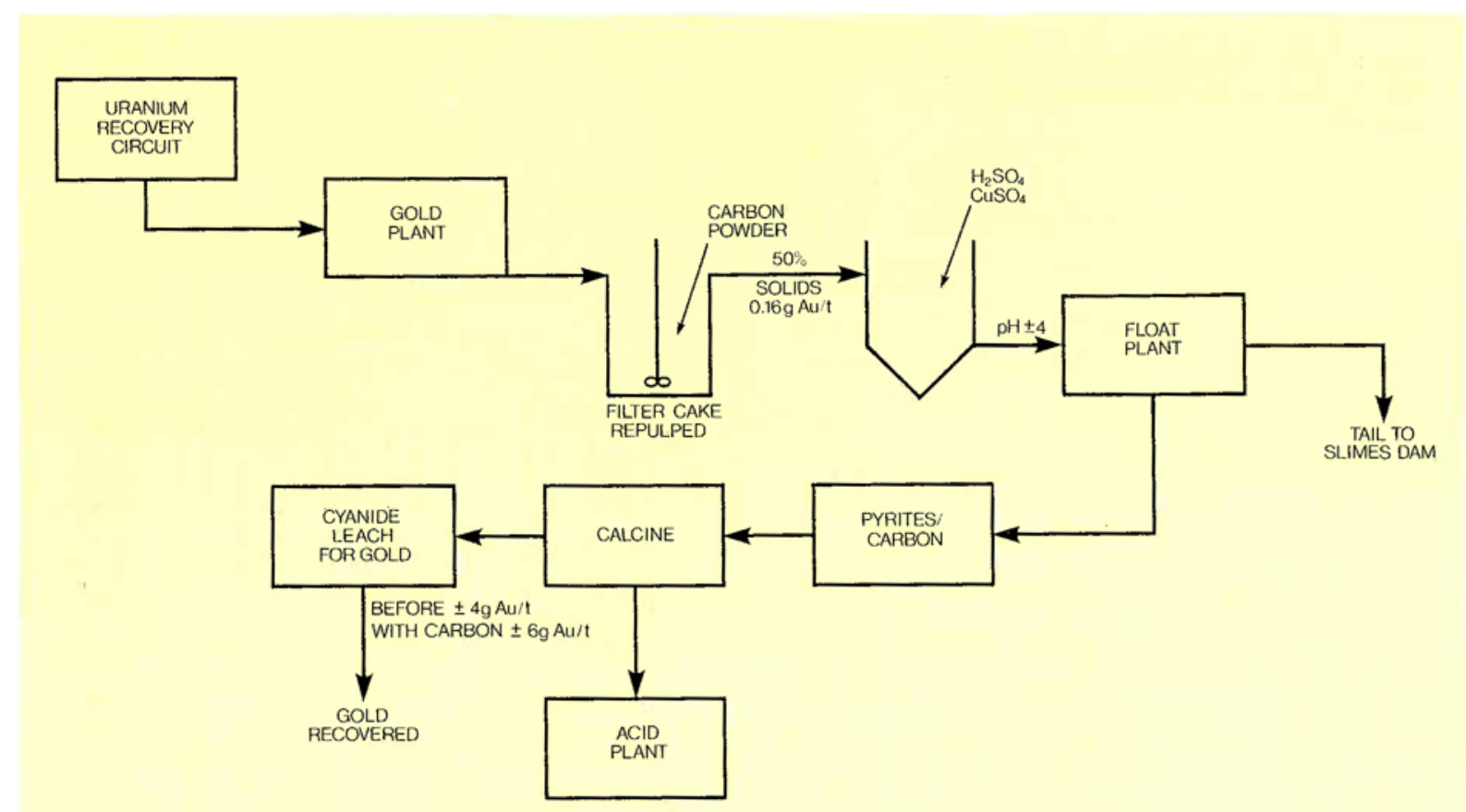

Fig. 2 Flow-sheet for a process in which the gold content of a calcine is upgraded by addition of activated carbon powder to the re-pulped filter cake

Secondly, fine carbon can be added to a cyanided pulp to adsorb gold $(9,15)$ and then be collected by a flotation technique. The gold can subsequently be recovered by burning the carbon. Plant trials on a reverse leach plant have shown that carbon powder can be added directly to the re-pulped filter cake to adsorb the soluble gold loss. The carbon powder is subsequently floated together with the pyrites and calcined. This results in a significant upgrading of the gold content (from 3.5 to $6 \mathrm{~g} / \mathrm{ton}$ ) of the calcine material. In this case, the fine carbon is floated together with the pyrites and calcined with it. A simplified flow-sheet for such a process is shown in Figure 2.

It is perhaps appropriate at this point to mention that it is improbable that carbon powder will ever be employed for the recovery of gold directly from pregnant cyanide liquors or pulps by a flotation technique. This is because the recovery of the fine carbon by flotation is not very efficient and high gold losses could be anticipated. Therefore, gold recovery processes that are based on a flotation technique will probably find application only in scavenging operations.

A further potentially important application for granular activated carbon is as a replacement for the zinc used in the Crowe-Merrill zinc dust precipitation circuit (16). Substitution of carbon for zinc dust would have many advantages, such as obviating the need to clarify and de-aerate the gold cyanide-containing filtrate and add excess cyanide to the filtrate.
Moreover, the carbon could be re-used after elution of the gold which could be recovered by electrowinning, whereas much of the zinc is lost by dissolution as $\mathrm{Zn}(\mathrm{CN})_{4}^{2-}$. Such an operation could also conveniently be conducted by a column technique.

Lastly, in cyanide heap leaching $(17,18)$ - a comparatively recent hydrometallurgical development for exploiting low grade ores, mine-waste material or deposits too small to justify the construction of milling facilities - activated carbon processes which offer a viable means of recovering the dissolved gold values have found application in the U.S.A.

In view of its high selectivity for gold (and silver) cyanide in the presence of large concentrations of base metals, and because it is relatively cheap, activated carbon is likely to play a significant future role in gold recovery processes such as those outlined above. Nevertheless, as will become apparent later in this review, there is at the moment no consensus of opinion on the mechanism by which activated carbon extracts the dicyanoaurate anion $\mathrm{Au}(\mathrm{CN})_{2}^{-}$from solution, and to date an empirical approach has had to be adopted in the selection of carbons for use in gold recovery processes. A complete understanding of the factors that govern the adsorption of gold cyanide on carbon and of the exact mechanism by which carbon loads gold is therefore of vital importance if the best use is to be made of this potentially important hydrometallurgical reaction. Further advances could lead either to the production of a carbon tailor-made for use in gold recovery processes - for example, a 
carbon with improved gold capacity and faster extraction kinetics - or to improvements in the current elution procedures. Thus, the development of a convenient and efficient room temperature elution procedure would be a significant improvement over the laborious current practice.

\section{Structure of Activated Carbon}

Activated carbon is a generic term for a family of substances none of which can be characterized by a definite structural formula or by chemical analysis. The various products are usually differentiated by their adsorptive properties. X-ray diffraction studies have shown that the structure of activated carbon is similar to that of graphite $(19,20)$. Ideal graphite, as shown in Figure 3, consists of layers of fused hexagons held approximately $0.335 \mathrm{~nm}$ apart by Van der Waals forces, so that the carbon atoms in any one plane lie above the centres of the hexagons in the layer immediately below it. The lattice is of the $A B A B$ type. The structure of activated carbon is depicted in Figure $4(19,20)$ and is believed to be composed of tiny graphite-like platelets which are only a few carbon atoms thick and 2 to $10 \mathrm{~nm}$ in

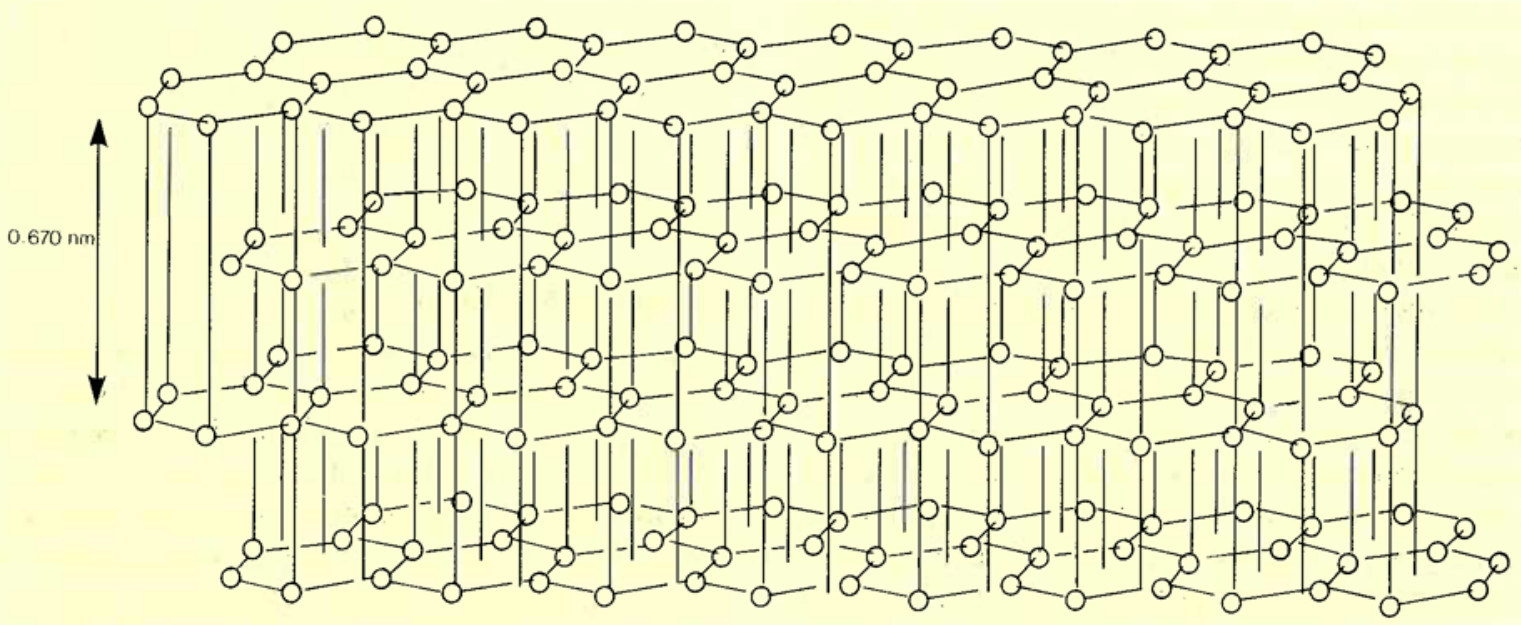

Fig.3 Schematic representation of the structure of graphite. The circles denote the positions of carbon atoms, whereas the horizontal lines represent carbon-to-carbon bonds

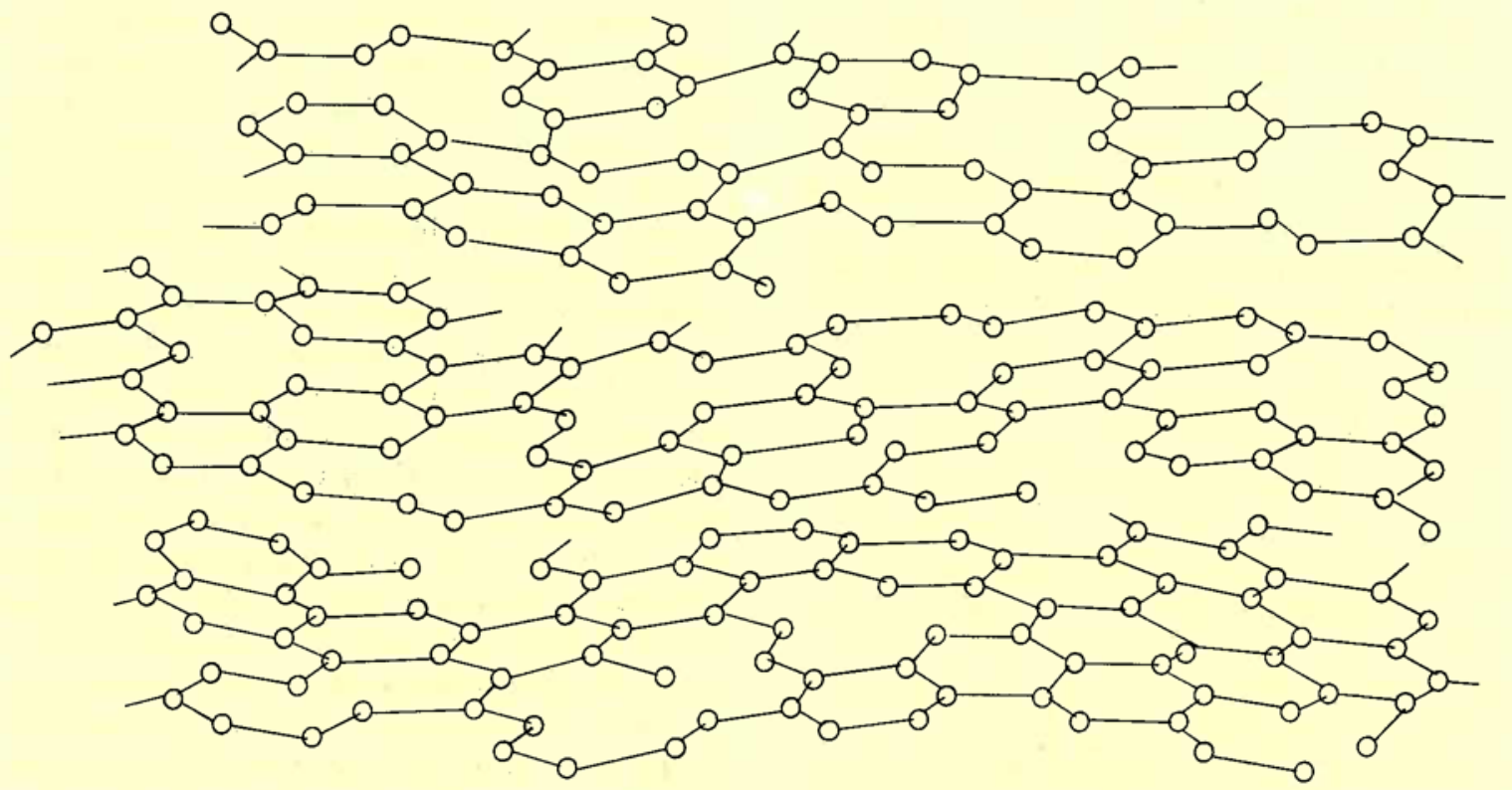

Fig. 4 Schematic representation of the proposed structure of activatéd carbon. Oxygen-containing organic functional groups are located at the edges of broken graphitic ring systems. After (20) 
diameter, and form the walls of open cavities of molecular dimensions. However, the hexagonal carbon rings, many of which have undergone cleavage, are randomly orientated. The overall structure is very disordered and often referred to as 'turbostratic'.

The process through which a pre-charred carbonaceous raw material (coal, peach pips, coconut shells etc.) develops a porous structure with an extended surface area $\left(600\right.$ to $1500 \mathrm{~m}^{2} / \mathrm{g}$ ), is referred to as activation (19), and is conducted at high temperature $\left(800\right.$ to $\left.1100^{\circ} \mathrm{C}\right)$ in the presence of a suitable oxidizing agent such as steam, carbon dioxide, air or any mixture of them. The available oxygen in the activating gas burns away the more reactive portions of the carbon skeleton as carbon monoxide and carbon dioxide, thereby increasing the surface area of the product and developing the pore structure. Taking cognizance of the many combinations of raw materials, and carbonization and activation conditions that can be employed in the manufacturing process, it is obvious that a great variety of carbons can be obtained.

The high level of structural imperfection in activated carbons results in many possibilities for reactions at the edge carbon atoms, so that the surface of activated carbon is composed primarily of oxygencontaining organic functional groups that are located mostly at the edges of broken graphitic ring systems. Although the identities of these groups are not known with certainty, those that have most often been suggested are:<smiles>C=Cc1cc(C(=O)O)c(C)c(C)c1C</smiles>

carboxyl groups

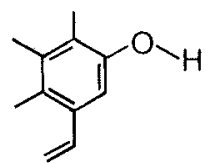

phenolic hydroxyl groups

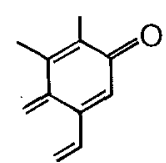

quinone-type carbonyl groups
Other suggestions relate to the presence of ether, peroxide and ester groups in the following forms:<smiles>Cc1ccc2oc(=O)c(C)c(C)c2c1C</smiles>

normal lactones<smiles>C=Cc1c(C)c(C)c(C=C)c2c1C(=O)OC2=O</smiles>

carboxylic acid anhydrides

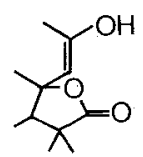

fluorescein-type lactones<smiles>c1ccc2c(c1)COOC2</smiles>

cyclic peroxides
Extensive discussions on the functional groups in activated carbon have been presented by Mattson and Mark (19), Boehm (21, 22) and Garten et al. (23).

\section{Interactions between Gold Complexes and Activated Carbon}

Although gold forms a large number of complexes with various ligands - thiourea, thiocyanate, cyanide, chloride, iodide, bromide, sulphide and thiosulphate among others - we are not aware of any work that has been published regarding the interactions between activated carbon and gold complexes other than those with chloride and cyanide which are of prime metallurgical interest. It should also be mentioned that although this review is not claimed to be an exhaustive appraisal of all the papers published in the field, it nevertheless covers all the theories that have been advanced over the years to account for the strong adsorption of gold cyanide on activated carbon.

\section{Chloride Medium}

Activated carbon interacts in a very versatile manner with inorganic species. In addition to being able to function as a simple adsorbent akin to polymeric adsorbents which load neutral organic molecules, it can also function as a reductant and under favourable conditions, for example in the presence of oxygen, as an oxidation catalyst. In fact, some carbons, especially those prepared by the high temperature steam activation route, have been shown to have a reduction potential of about $-0,14 \mathrm{~V}$ against the saturated calomel electrode (SCE) as measured by a graphite rod technique (24). Therefore, it is not suprising that with the gold chloride complex $\mathrm{Au}(\mathrm{Cl})_{4}^{-}$, for the reduction of which the potential $\mathrm{E}^{\circ}$ is $+0.8 \mathrm{~V}$ against SCE, reduction by certain carbons to metallic gold occurs readily, as first observed by Brussov (25). Reduction proceeds by transfer of electrons from the interior to the surface of the carbon granule and the gold, even at relatively low loadings, is visible on the surface of the carbon (Figure 5). In this case, there is no difficulty in deciding on the adsorption mechanism. Similarly, gold complexes such as $\mathrm{AuBr}_{2}^{-}$ and $\mathrm{AuI}_{2}^{-}$which have $\mathrm{E}^{\circ}$ values of +0.7 and $+0.3 \mathrm{~V}$ against SCE respectively, are loaded partially or completely by a reduction mechanism on activated carbons which have lower $\mathrm{E}^{\circ}$ values, such as the $-0.14 \mathrm{~V}$ mentioned above. The complex is reduced to metallic gold which is retained on the external surface of the carbon granules by Van der Waals forces. On the other hand, the cyanide ion forms very strong complexes with gold $\left(\log \beta_{2}=38\right)$, and a potential of $-0.85 \mathrm{~V}$ against SCE is required to reduce $\mathrm{Au}(\mathrm{CN})_{2}^{-}$ to metallic gold. Therefore, it is generally regarded as 
most improbable that activated carbon loads gold cyanide by complete reduction of the complex to metallic gold, as is observed with the chloride, bromide and iodide complexes.

\section{Cyanide Medium}

The mechanism by which activated carbon loads gold cyanide $\mathrm{Au}(\mathrm{CN})_{2}^{-}$has interested and puzzled researchers since 1913, but as this article goes to press, still remains a matter of much controversy.

\section{Early Theories}

Earlier theories 'on the mechanism of adsorption can be divided into essentially two types. First, those that proposed that the $\mathrm{Au}(\mathrm{CN})_{2}^{-}$anions are adsorbed as such. Secondly, those that proposed that the gold cyanide complex is altered chemically by reduction to metallic gold or to some intermediate state, or by precipitation as the insoluble complex $\mathrm{AuCN}$ in the pores of the carbon.

In 1913, Green (26) published the results of the first investigation into the so-called precipitation of gold cyanide onto finely crushed wood charcoal. He claimed that the wood charcoal turned a yellow colour after contact with a gold cyanide solution and proposed that the loading mechanism involved the complete reduction of the cyanide complex to metallic gold. (It should be mentioned that Green is the only researcher to have claimed seeing a change in the colour of the carbon on loading it from cyanide solution.) In degassing experiments on a freshly prepared wood charcoal, Green established that it contained two types of gases, namely $12 \mathrm{~cm}^{3} / \mathrm{g}$ of a mixture of loosely held gases $\left(18 \mathrm{O}_{2} / 20 \mathrm{CO} / 37 \mathrm{H}_{2} / 26 \mathrm{~N}_{2}\right.$ volume per cent) that were readily expelled at room temperature under vacuum and $250 \mathrm{~cm}^{3} / \mathrm{g}$ of a mixture of strongly held gases $\left(12 \mathrm{CO}_{2} / 26 \mathrm{CO} / 58 \mathrm{~N}_{2} / 1 \quad \mathrm{H}_{2}\right.$ volume per cent) which were expelled only at high temperature. He demonstrated that charcoal from which the adsorbed gases had been removed at $500^{\circ} \mathrm{C}$, was a poor precipitant for gold and suggested that the ability to load gold cyanide was associated with the presence of occluded reducing gases in the charcoal, notably carbon monoxide (only a small amount of hydrogen gas had been detected in the carbon). Since carbon monoxide (or hydrogen) passed through a gold cyanide solution did not precipitate metallic gold, Green advanced the hypothesis that the occluded carbon monoxide was in a highly condensed, and hence active, state on the carbon surface. He also showed that pure graphite did not load gold from cyanide solutions.

The theories of Green were later quite validly criticised in a contribution to the discussion of Edmand's paper by Allen (27) who suggested that the carbon monoxide and dioxide liberated on heating to $500^{\circ} \mathrm{C}$ originated in the destruction of oxygencontaining organic functional groups on the carbon surface and not in the presence of adsorbed carbon monoxide molecules on the surface of the carbon. Continuing his argument, Allen stated that it was most unlikely that a gas that was liberated only at $500^{\circ} \mathrm{C}$ could be responsible for the gold precipitation reaction that occurred readily at room temperature.

Although, as previously mentioned, the identities of the oxygen-containing organic functional groups that are formed on the surface of carbon during its activation are not known with certainty, one of the important roles of these groups undoubtedly is to impart a hydrophilic character to the predominantly hydrophobic skeleton of the carbon. Since, as pointed out by Allen (27) and subsequently confirmed by other researchers (19), heating the carbon to a high temperature under vacuum results in the destruction of these groups with the liberation of carbon monoxide and carbon dioxide, the inability of carbons so treated to adsorb gold cyanide might simply be related to failure of the gold cyanide solution to wet and make contact with the now hydrophobic surface. However, soon afterwards, Feldtmann (28) found that although metallic gold precipitated rapidly from chloride medium onto 10 mesh wood charcoal, in a form that was readily soluble in a weak cyanide solution, the gold in an aurocyanide solution was loaded onto carbon comparatively slowly, in a form that was neither visibly metallic nor to any significant extent soluble in cyanide solutions, thereby contradicting Green's (26) experiments in part. From these results it was concluded that the action of charcoal on gold cyanide was quite different from that on gold chloride.

The factors that influence the loading of gold cyanide on charcoal were also investigated by Feldtmann, and the following conclusions were drawn:

(1) Increasing free cyanide concentration depressed gold loading

(2) Increasing free hydroxide concentration had a negligible effect on gold capacity

(3) The absolute amount of gold loaded on charcoal increased with the gold concentration, although the overall percentage extraction decreased with that variable

(4) The rate of gold loading on charcoal was slow and equilibrium was not reached in 48 hours.

A serious criticism of these experiments, however, is that they were conducted without suitable agitation of the carbon-solution mixture. With regard to the elution of gold cyanide from charcoal, Feldtmann found that the gold was soluble at room temperature in solutions of alkaline sulphides such as $\mathrm{H}_{2} \mathrm{~S}, \mathrm{~K}_{2} \mathrm{~S}$, $\mathrm{Na}_{2} \mathrm{~S}$, KHS and NaHS. However, ammonium 
sulphide and alkaline polysulphides were found to be less effective eluants.

Feldtmann also established that cyanogen gas formed and was retained by the charcoal during exposure to gold cyanide solutions containing excess potassium cyanide. The explanation advanced for the formation of the gas involved the oxidation of hydrogen cyanide (generated by hydrolysis) by oxygen occluded in the charcoal according to:

$$
2 \mathrm{HCN}+1 / 2 \mathrm{O}_{2} \rightleftharpoons(\mathrm{CN})_{2}+\mathrm{H}_{2} \mathrm{O}
$$

The same author postulated that gold cyanide was loaded on charcoal not in the metallic or colloidal metallic state, but by a chemical precipitation mechanism involving a combination of $\mathrm{AuCN}, \mathrm{CO}$ and $(\mathrm{CN})_{2}$, that is a 'carbonyl aurocyanide' precipitate. Adopting as a working hypothesis the formula AuCN.CO $(\mathrm{CN})_{2}$ for the gold adsorbate, the successful elution of this compound from the charcoal with the alkaline sulphide eluant was attributed to the reaction:

$$
\begin{aligned}
\mathrm{AuCN} \cdot \mathrm{CO}(\mathrm{CN})_{2}+\mathrm{Na}_{2} \mathrm{~S} \rightleftharpoons & \underset{\mathrm{NaAu}(\mathrm{CN})_{2}+\mathrm{NaSCN}+\mathrm{CO}}{ }
\end{aligned}
$$

because Feldtmann was unable to detect aurosulphide $\mathrm{AuS}^{-}$in solution $\mathrm{AuS}^{-}$is precipitated from solution with $\mathrm{MgCO}_{3}$ ) and also because the eluate contained a considerable amount of thiocyanate. The readsorption of the carbon monoxide produced in equation (2) was believed to restore the gold activity of the charcoal. (It is a generally observed phenomenon that, in the absence of organic fouling, activated carbon can undergo many loading and elution cycles without losing its capacity for the aurocyanide ion.)

Picard (28), in a contribution to a discussion of Feldtmann's work, then suggested an alternative mechanism to explain the latter's results. This was based on the fact that certain processes such as oxidation-filtration and bleaching of solutions containing organic matter, depend on the oxidizing power of charcoal (imparted to it by adsorbed oxygen), so that oxidation might similarly be responsible for gold cyanide precipitation on charcoal. His mechanism involved the precipitation of insoluble aurous cyanide AuCN in the pores of the carbon, accompanied by the formation of a cyanate in accordance with the equation:

$$
\mathrm{KAu}(\mathrm{CN})_{2}+1 / 2 \mathrm{O}_{2} \rightleftharpoons \mathrm{AuCN}+\mathrm{KCNO}
$$

Feldtmann (28) also investigated West African graphitic schists which were sometimes encountered with certain gold ores and were a nuisance, because they extracted gold cyanide. However, it is doubtful whether the carbonaceous matter in the schists was true graphite, because Green (26) and others (29) have shown that pure graphite or graphitic oxide has no gold cyanide activity, and it is likely that the material in the schists was akin to activated carbon.

The announcement in 1.916 that charcoal was to replace zinc as a precipitant for gold cyanide at the Yuanmi Mine in Australia gave new impetus to research and renewed efforts were made to resolve the loading mechanism. Although Edmands (27) confirmed many of the results obtained by Feldtmann (28), he disagreed on the details of the mechanism proposed by him. In view of the tendency of the potassium dicyanoaurate complex $\mathrm{KAu}(\mathrm{CN})_{2}$ to form with the halogens, addition products of general formula $\mathrm{KAu}(\mathrm{CN})_{2}(\mathrm{X})_{2}$, where $\mathrm{X}$ can be $\mathrm{Cl}^{-}, \mathrm{Br}^{-}$or $\mathrm{I}^{-}$, Edmands, still favouring the idea that carbon monoxide was somehow involved, suggested that the carbon monoxide occluded in the charcoal formed an analogous addition compound with gold cyanide, namely $\mathrm{KAu}(\mathrm{CN})_{2} \mathrm{CO}$, or $\mathrm{HAu}(\mathrm{CN})_{2} \mathrm{CO}$ in the case of loading from acidified cyanide solutions. Thus, the mechanism suggested by Edmands involved the oxidation of $\mathrm{Au}(\mathrm{I})$ to $\mathrm{Au}(\mathrm{III})$.

The effect of the presence of various compounds in the solution on the ability of charcoal to load gold cyanide was also investigated. Edmands found that the gold capacity of charcoal was greatly enhanced in acidic solution, whereas soluble sulphides, as good eluants, depressed it. On the other hand, the addition of thiocyanate and thiosulphate to the solution was found to have a negligible effect on the gold capacity. The same author also found that the time required to reach equilibrium varied with agitation, the particle size of the charcoal, and the relative amounts of charcoal and gold present in the solution. Aeration of the solution was found to have no effect on the gold capacity, in contrast to what has been reported by later workers (38).

So far, the word 'charcoal' has been used deliberately instead of activated carbon, because in the early days very little was known about the preparation of high surface area activated carbons. Moreover, the charcoals prepared by the earlier workers by bringing wood chunks to red heat and then quenching in water, were undoubtedly not very active. These products probably had low surface areas coupled with poorly developed pore systems and it would be technically incorrect to refer to them as activated carbon. These remarks are partly supported by the work of Feldtmann (27), who in a contribution to a discussion of Edmands' results stated that he had found that the gold(III) cyanide complex $\mathrm{KAu}(\mathrm{CN})_{4}$ was not loaded by charcoal. On modern carbons, $\mathrm{Au}(\mathrm{CN})_{4}^{-}$and $\mathrm{Au}(\mathrm{CN})_{2}^{-}$are loaded equally well, and it would appear as though the pore structure of the early 
Fig. 5 Three types of activated carbon particles coated with metallic gold after loading from $\mathrm{Au}\left(\mathrm{Cl}_{4}\right)^{-}$solutions. The average size of the particles is approximately $2 \mathrm{~mm}$ in all three cases

Peach pip carbon particles

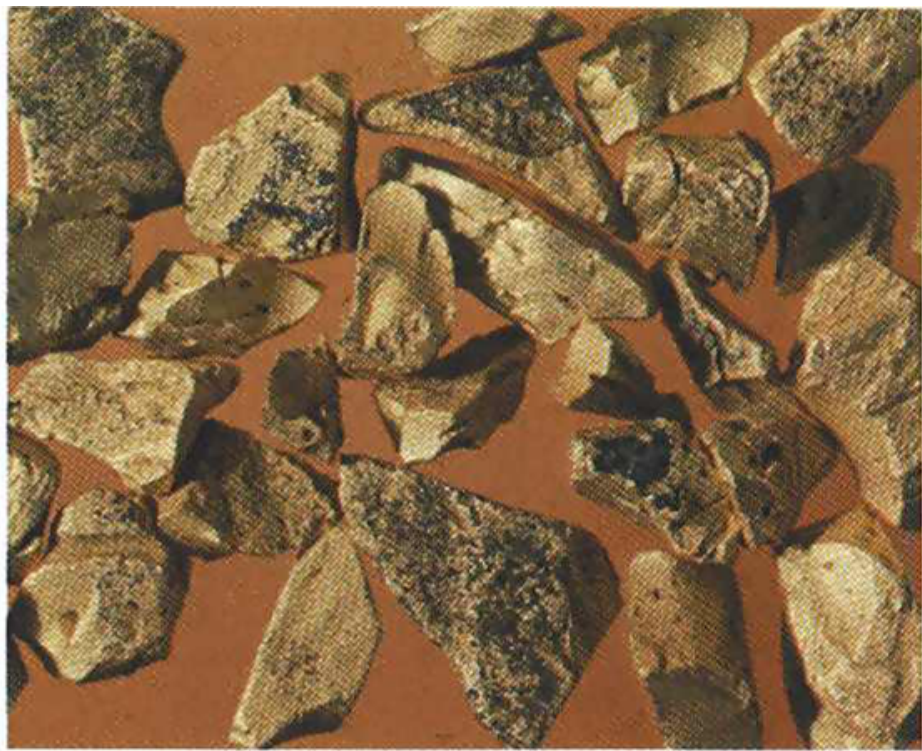

Carbon spheres derived from polystyrene beads, including some (black) which have not been exposed to the gold(III) chloride solution
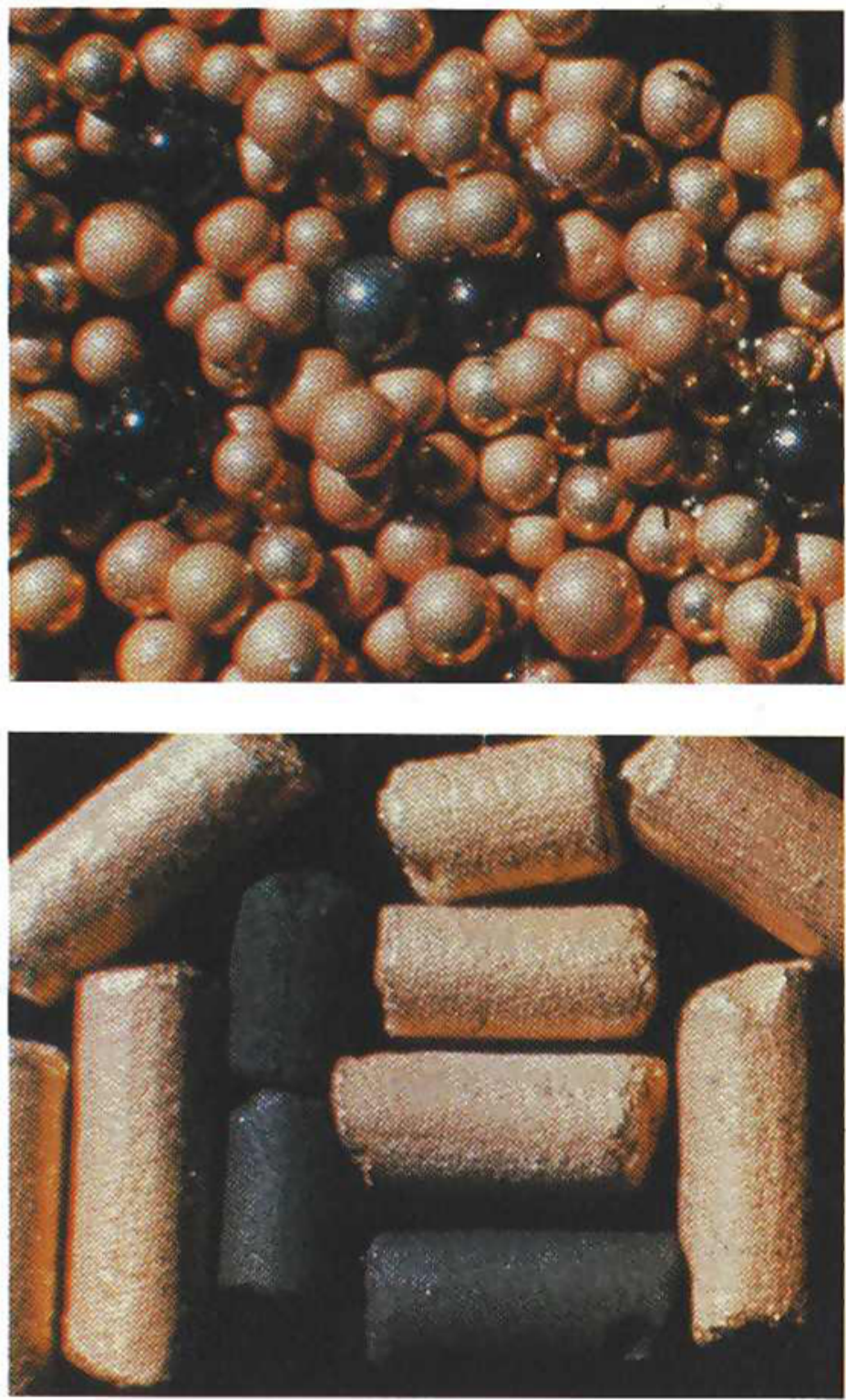

Extruded peat-based carbon pellets 
wood charcoals was not sufficiently developed to permit entry of the square planar gold(III) complex whereas the linear gold(I) complex could penetrate the interior of the charcoal. Thus, the negligible loading of $\mathrm{Au}(\mathrm{CN})_{4}$ on early charcoal probably reflected a sieve or screening effect, rather than chemical considerations.

The assumption that some chemical reaction took place between carbon monoxide gas, believed to be occluded by carbon, and gold cyanide, whereby a carbonyl aurocyanide or other analogous compound was formed, was widely accepted by early workers, However, this theory fell into disfavour when Allen, in a contribution to a discussion of Edmands' paper (27), criticised the popular theories of those times and proposed that gold cyanide was physically adsorbed as $\mathrm{NaAu}(\mathrm{CN})_{2}$ on charcoal without undergoing chemical change (4).

Allen advanced his theory when he found that Feldtmann's (28), results for the loading of gold cyanide from synthetic solution onto charcoal could be accurately described by the empirical Freundlich equation, which in the case of true adsorption phenomena, relates the amount of material adsorbed to the concentration of solute remaining in solution at equilibrium. The mathematical form of the Freundlich isotherm is:

$$
\frac{\mathrm{x}}{\mathrm{m}}=\mathrm{kc} \mathrm{c}^{\mathrm{in}}
$$

where:

$\mathrm{x}$ is the mass of the adsorbate

$m$ is the mass of the adsorbent

$c$ is the equilibrium concentration of solute in the solution

$\mathrm{k}$ and $\mathrm{n}$ are constants for a particular solute.

Allen's conclusions were generally accepted for several years, until Williams (30) showed that the sodium in the ash of the loaded, burned charcoal from the Yuanmi Mine was far from being sufficient to account for the simple adsorption of $\mathrm{NaAu}(\mathrm{CN})_{2}$ from solution, and that it was more likely to be the anion $\mathrm{Au}(\mathrm{CN})_{2}$ that was loaded. Williams recognised the fact that the ash content of the charcoal might play a significant role in the adsorption mechanism and suggested that further research could profitably be carried out on the adsorption of gold onto more uniform charcoals from solutions containing only $\mathrm{Au}(\mathrm{CN})_{2}^{-}$as opposed to gold plant liquors that also contained a variety of other species.

By 1921, owing largely to the work of Ardagh (31), knowledge of the actual preparation of active carbons had significantly advanced. Ardagh advocated what is now the basis of modern activation procedures, namely that the wood should be heated to a high temperature in the presence of steam to decompose and expel inactive hydrocarbon material (or other oxidizing gases such as carbon dioxide), if high adsorptive power for gases was desired. However, it was realized at the time (30) that this activation process would probably also have an important effect on the activity of carbons for the adsorption of gold cyanide from solution.

This was the position when, in the late 1920s, Gross and Scott (32) of the United States Bureau of Mines Laboratories, undertook the first systematic and detailed investigation of the factors that influence the adsorption onto activated carbon of $\mathrm{Au}(\mathrm{CN})_{2}^{-}$ from synthetic solutions. The objectives of the study were to elucidate the exact mechanism by which carbon extracted gold cyanide, and also to investigate potential commercial applications of activated carbon in the gold mining industry. Significant findings regarding the adsorption of $\mathrm{Au}(\mathrm{CN})_{2}^{-}$on a pine wood carbon that had been activated with steam at $900^{\circ} \mathrm{C}$ for 30 minutes were:

(1) The rate of gold extraction was slow (equilibrium was not reached in 24 hours), and a state of reversible equilibrium was established between the gold on the carbon and the gold remaining in solution

(2) The maximum capacity of the carbon for gold was 7 per cent by mass, which assuming monomolecular adsorption (based on the theories of Langmuir), as well as a surface area of $230 \mathrm{~m}^{2} / \mathrm{g}$ for the carbon (modern carbons have a surface area of about $1000 \mathrm{~m}^{2} / \mathrm{g}$ ), corresponded to a surface coverage of only 23 per cent

(3) Silver cyanide $\mathrm{Ag}(\mathrm{CN})_{2}^{-}$displaced 15.3 per cent of an equilibrium amount of adsorbed gold, whereas $\mathrm{Au}(\mathrm{CN})_{2}^{-}$displaced 68.3 per cent of an equilibrium silver loading

(4) Although acids were known at the time to depress precipitation of metallic gold onto carbon from $\mathrm{Au}(\mathrm{Cl})_{4}^{-}$solutions, acidification of gold cyanide solutions enhanced significantly the gold capacity of the carbon, as was to be observed by many other later researchers

(5) Excess free hydroxide, sulphide and cyanide depressed the gold capacity, in agreement with the results of others (28). This led to the suggestion that these substances should be good eluants for the adsorbed gold $\left(\mathrm{CN}^{-}\right.$and $\mathrm{OH}^{-}$are currently employed in elution procedures)

(6) Although alkaline sulphides were excellent eluants for the gold that was adsorbed onto carbon from cyanide solution, they were poor eluants for the metallic gold that was precipitated onto carbon from chloride medium. This supported the authors' view that the mechanisms of adsorption from these two types of solutions were fundamentally different 
(7) The maximum capacity of the carbon for adsorption of gold from chloride solutions was significantly higher than that from cyanide solutions, namely 569 against $68 \mathrm{~kg}$ of gold per ton of carbon

(8) The capacity of the carbon for gold from cyanide solution decreased as the temperature of the solution was increased. This was attributed to the higher solubility of gold salts in hot water than in cold water. (It is generally known that if two species in a loading solution are competing for adsorption onto carbon, the carbon will prefer that which is least soluble.)

Analyses of solutions from which gold as $\mathrm{KAu}(\mathrm{CN})_{2}$ was loaded onto activated pine wood and sugar carbons, revealed that the solutions contained a large amount of bicarbonate and that the potassium ion remained in solution. This confirmed the work of Williams (30) who had been unable to find a sufficient amount of sodium in ashed carbon to account for the loading of $\mathrm{NaAu}(\mathrm{CN})_{2}$ as such from solution. Gross and Scott suggested

$$
\begin{aligned}
2 \mathrm{KAu}(\mathrm{CN})_{2}+\mathrm{Ca}(\mathrm{OH})_{2}+2 \mathrm{CO}_{2} \rightleftharpoons \\
\mathrm{Ca}\left[\mathrm{Au}(\mathrm{CN})_{2}\right]_{2}+2 \mathrm{KHCO}_{3}(5)
\end{aligned}
$$

as the mechanism for the adsorption of gold cyanide onto activated pine wood carbon which was found to contain $\mathrm{Ca}(\mathrm{OH})_{2}$ (50 per cent of the ash of burned pine carbon was found to be $\mathrm{CaO}$ ). This mechanism was based on a chemical interaction between calcium ions present in the ash content of the carbon and the $\mathrm{Au}(\mathrm{CN})_{2}^{-}$anions, with the cation of the gold complex remaining in solution as part of a bicarbonate complex which was formed with the carbon dioxide occluded in the carbon.

On the other hand, for a sugar carbon that contained no metal ions (the ash content was only 0.25 per cent), a different mechanism was proposed:

$$
\mathrm{KAu}(\mathrm{CN})_{2}+\mathrm{H}_{2} \mathrm{O}+\mathrm{CO}_{2} \rightleftharpoons \mathrm{HAu}(\mathrm{CN})_{2}+\mathrm{KHCO}_{3}(6)
$$

In this case it was assumed that water molecules were dissociated into $\mathrm{H}^{+}$and $\mathrm{OH}^{-}$ions in the carbon, thereby supplying the cations necessary to preserve the charge balance. For adsorption from alkaline solution, Gross and Scott therefore proposed that the $\mathrm{Au}(\mathrm{CN})_{2}^{-}$anions were loaded without undergoing a chemical change and retained in the carbon as a neutral complex, namely $\mathrm{M}^{\mathrm{n}+}\left[\mathrm{Au}(\mathrm{CN})_{2}^{-}\right]_{\mathrm{n}}$ where the identity of $\mathrm{M}^{\mathrm{n}+}$ depended on the nature of the metals present in the ash content of the carbon.

The enhanced gold loading in acidic solutions was attributed to the formation of insoluble AuCN:

$$
\mathrm{Au}(\mathrm{CN})_{2}^{-}+\mathrm{H}^{+} \rightleftharpoons \mathrm{AuCN}+\mathrm{HCN}
$$

This AuCN was precipitated and retained in the pores of the carbon. Therefore, the difference in the capacities of carbon for gold from acidic and alkaline solutions was attributed to precipitation in the former and adsorption in the latter.

Gross and Scott claimed that free $\mathrm{CN}^{-}$anions were irreversibly adsorbed on carbon from a solution of potassium cyanide. However, it is now well established that activated carbon has a very low affinity for small, highly hydrated inorganic ions, and it is more likely that the carbon which is an oxidation catalyst, simply oxidized the $\mathrm{CN}^{-}$anions, thereby making it appear that they had been irreversibly adsorbed. In fact, activated carbon is currently being employed in processes requiring catalytic destruction of $\mathrm{CN}^{-}$(33).

The same authors also investigated the loading of gold onto carbons prepared under different conditions from various raw materials, and their results indicated that the carbons that were activated with steam at high temperature had the best gold activity.

The mechanisms that were advanced by Allen (27), Williams (30), and Gross and Scott (32) were in agreement regarding the adsorption of $\mathrm{Au}(\mathrm{CN})_{2}^{-}$anions on carbon without the occurrence of a chemical change and differed only with respect to the source of the cation required to maintain the charge balance.

\section{Modern Theories}

The work discussed in the previous section was followed by numerous publications proposing that $\mathrm{Au}(\mathrm{CN})_{2}^{-}$is adsorbed on activated carbon without being decomposed. Garten and Weiss (34) in 1957, offered an alternative explanation for the experimental results of Gross and Scott (32) and suggested that $\mathrm{Au}(\mathrm{CN})_{2}^{-}$anions were loaded on carbon by an anionexchange mechanism involving simple electrostatic interactions between positive and negative charges. The positive sites on the carbon were assumed to be carbonium ion sites, the formation of which in alkaline solution was attributed to the oxidation of chromene groups that were assumed to exist on the carbon surface, to chromenol groups by the oxygen adsorbed on the surface of the carbon:

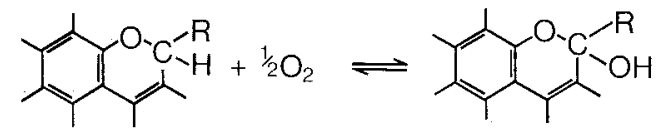

Thus, it was suggested that $\mathrm{CN}^{-}$and $\mathrm{Au}(\mathrm{CN})_{2}^{-}$ anions could be exchanged for the $\mathrm{OH}^{-}$anions of the chromenol groups in accordance with:

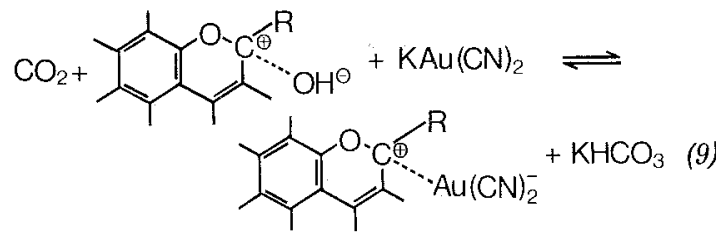


However, Garten and Weiss did not offer an explanation for the enhanced loading which is observed from acidified cyanide solutions.

In contrast, Kuzminykh and Tjurin (35) quite validly argued that since the presence of simple anions in the cyanide solution, for example $\mathrm{Cl}^{-}$or $\mathrm{I}^{-}$ even at concentrations as high as $1.5 \mathrm{M}$, had no effect on the capacity of birch or aspen carbons for gold cyanide, the interactions between gold cyanide and carbon could not be electrostatic in nature. Furthermore, since neutral organic molecules, for example octyl alcohol and kerosene, if present together with gold cyanide in solution were found to depress the gold capacity of the carbon, these authors proposed that the gold adsorbate was a neutral molecule, the nature of which depended on the $\mathrm{pH}$ of the solution. Thus, under acidic conditions they suggested that neutral molecules of $\mathrm{HAu}(\mathrm{CN})_{2}$ which is a strong acid and is therefore completely dissociated in solution, were concentrated on the surface of the carbon by a capillary condensation mechanism, thereby acquiring the properties of a typical molecular sorbate. (It is known that carbon has greater affinity for neutral molecules than for charged species.) Under neutral or alkaline conditions, a salt such as $\mathrm{NaAu}(\mathrm{CN})_{2}$ was adsorbed. The gold complexes were believed to be held on the carbon by 'dispersion forces', that is by Van der Waals-type interactions.

Kuzminykh et al. (36) also discounted the mechanism proposed by Feldtmann for the elution of adsorbed gold cyanide with alkaline sulphide solutions (see equation (2)) and proposed instead that the gold was desorbed in the form of what they termed 'adsorptively inactive' aurous sulphide $\mathrm{Au}_{2} \mathrm{~S}$. This mechanism is very unlikely, since $\mathrm{Au}_{2} \mathrm{~S}$ is highly insoluble in aqueous medium and, if formed, would tend to be retained in the pores of the carbon as a solid precipitate rather than be desorbed. Since Feldtmann (28) has shown that the mechanism of desorption with sulphide ions does not involve the formation of $\mathrm{AuS}^{-}$anions, and also because it is unlikely that simple exchange of $\mathrm{Au}(\mathrm{CN})_{2}^{-}$for $\mathrm{S}^{2-}$ anions occurs, the mechanism by which sulphide anions are able to elute $\mathrm{Au}(\mathrm{CN})_{2}^{-}$from carbon remains unknown.

Davidson (37) investigated the factors that influence the adsorption of $\mathrm{Au}(\mathrm{CN})_{2}^{-}$on activated coconut shell carbon, and found that reproducible isotherms could be obtained only in the presence of a borate buffer solution. The main emphasis of his work was towards the influence of 'spectator cations', for example $\mathrm{Ca}^{2+}$ and $\mathrm{Na}^{+}$that were added to the adsorption medium, and of temperature on the gold capacity of the carbon. He found, as earlier and later workers did, that the presence of acid promoted gold loading, as well as the hitherto unknown fact that the presence of salts in solution, for example $\mathrm{CaCl}_{2}$ and $\mathrm{NaCl}$, enhanced the gold capacity of the carbon. This latter point has recently been confirmed. The mechanism advanced by Davidson to account for his experimental observations involved the adsorption of the metal dicyanoaurate complex ion-pair $\mathrm{M}^{\mathrm{n}+}\left[\mathrm{Au}(\mathrm{CN})_{2}^{-}\right]_{\mathrm{n}}$ from solution. Why these salts should be adsorbed on the carbon surface was not explained, but it was noted that the ability of $\mathrm{M}^{\mathrm{n}+}$ to inhibit gold desorption with alkaline carbonate solutions followed the sequence:

$$
\mathrm{Ca}^{2+}>\mathrm{Mg}^{2+}>\mathrm{H}^{+}>\mathrm{Li}^{+}>\mathrm{Na}^{+}>\mathrm{K}^{+}
$$

These observations led to the conclusion that when $\mathrm{M}^{\mathrm{n}+}$ was an alkaline earth metal ion $\left(\mathrm{Ca}^{2+}\right.$ or $\left.\mathrm{Mg}^{2+}\right)$ the ion-pair was bound to the carbon more firmly than when $\mathrm{M}^{\mathrm{n}+}$ was an alkali metal ion $\left(\mathrm{Na}^{+}\right.$or $\left.\mathrm{K}^{+}\right)$.

The practical implication of this conclusion was that elution procedures should involve a pretreatment, with $\mathrm{K}_{2} \mathrm{CO}_{3}$ for example, so as to ion-exchange the $\mathrm{Ca}^{2+}$ cation with $\mathrm{K}^{+}$according to:

$$
\mathrm{Ca}\left[\mathrm{Au}(\mathrm{CN})_{2}\right]_{2}+\mathrm{K}_{2} \mathrm{CO}_{3} \rightleftharpoons 2 \mathrm{~K}\left[\mathrm{Au}(\mathrm{CN})_{2}\right]+\cdot \mathrm{CaCO}_{3}(10)
$$

because this would convert the firmly bound ion-pair into a more readily desorbable species. However, as pointed out by Cho (48), enhancement of the desorbtion of gold from carbon in this manner does not necessarily imply that the replacement of calcium with potassium is the primary driving force for the observed desorption effect, since Gross and Scott (32) showed that the best eluants for gold cyanide were those substances that raised the $\mathrm{pH}$ of the solution $(\mathrm{NaOH}$ and $\mathrm{NaCN})$. Cho concluded that the fact that solutions of carbonates in water are alkaline could equally well account for the observations reported by Davidson.

Dixon, Cho and Pitt (38) found that the rate of adsorption of gold cyanide on activated coconut shell carbon was controlled by pore diffusion in the temperature range 25 to $55^{\circ} \mathrm{C}$ and for gold concentrations of 100 to $200 \mathrm{mg} / 1$, with an activation energy of 8 to $13 \mathrm{~kJ} / \mathrm{mol}$. They also found that the size of the carbon granules affected only the initial rate of adsorption, observing faster rates for the smaller size fractions, and that the equilibrium gold capacity was unaffected. In agreement with the results of other investigators $(24,37)$, the same authors observed that the equilibrium gold loading is strongly dependent on the $\mathrm{pH}$ value of the solution - the amount of gold adsorbed in the $\mathrm{pH}$ range 4 to 7 being about twice that adsorbed in the pH range 8 to 11 .

The adsorption mechanism advanced by Dixon et al. involved an electrostatic interaction between $\mathrm{Au}(\mathrm{CN})_{2}^{-}$anions and positively charged sites on the 
surface of the carbon, as previously suggested by Plaksin (39), and Garten and Weiss (34). However, they postulated that the sites were formed in accordance with the theories of Frumkin et al. $(40,41,42)$ which were accepted as accounting for the adsorption of acid on carbon. According to Frumkin's electrochemical model, oxygen in contact with an aqueous slurry of carbon is reduced to hydroxyl ions, with the liberation of hydrogen peroxide as follows:

$$
\mathrm{C}+\mathrm{O}_{2}+2 \mathrm{H}_{2} \mathrm{O} \rightleftharpoons \mathrm{C}^{2+}+2 \mathrm{OH}^{-}+\mathrm{H}_{2} \mathrm{O}_{2}
$$

Since the carbon supplies the electrons, it acquires a positive charge which, in order to maintain electrical neutrality, attracts anions such as $\mathrm{Au}(\mathrm{CN})_{2}^{\text {- }}$.

The reaction shown in equation (11) was also suggested as responsible for the higher gold loading observed in acidic solutions. By Le Chatelier's Principle, it would be expected that at low $\mathrm{pH}$ value the equilibrium would be shifted towards the right, thereby producing more positively charged sites that would attract an equivalent number of $\mathrm{Au}(\mathrm{CN})_{2}^{-}$ anions. Contrary to Edmands (27), Dixon et al. found that the gold capacity of carbon was enhanced when molecular oxygen was passed through the solution, whereas it was not when nitrogen was bubbled, an observation that could also be explained in terms of equation (11). Furthermore, they showed that the gold capacity of the carbon is inversely related to temperature.

On the basis of Davidson's results (37) which indicated that a loading of $70 \mathrm{mg}$ gold per gram of carbon was in equilibrium with a gold concentration in solution of $30 \mathrm{mg} / 1$ for a coconut shell carbon of specific surface area $1000 \mathrm{~m}^{2} / \mathrm{g}$, Clauss and Weiss (29) calculated that the surface coverage amounted to less than one gold atom per $5 \mathrm{~nm}^{2}$ of available carbon surface. This low figure led them to suggest that gold cyanide adsorption occurred as a result of specific interactions between the $\mathrm{Au}(\mathrm{CN})_{2}^{-}$anions and preferred sites on the carbon surface, as opposed to a general adsorption over the whole surface. The observation that the gold activity of the carbon could be completely destroyed by subjecting the carbon to certain chemical pretreatments, for example boiling in a $1 / 1$ mixture of concentrated nitric and sulphuric acids at $80^{\circ} \mathrm{C}$ for 2 hours, was advanced as proof of their theory. On the basis of similar experiments, they excluded basal planes, carboxylic acid groups and basic oxides as so-called 'points of attachment'. Addition of an oxidizing agent to the solution, for example hydrogen peroxide, was found to enhance the gold activity of the carbon, whereas reductants, for example hydrazine and hydroquinone, had the opposite effect. These observations were regarded as an indication that the adsorption sites were probably quinone- type groups. However, as an alternative suggestion, Clauss and Weiss pointed out that a special type of micropore might be responsible for gold adsorption. They also found that pure graphite (26), as well as graphitic oxide, did not load gold cyanide.

This work is subject to numerous criticisms. Carbon is attacked by hot concentrated sulphuric acid and especially by hot concentrated nitric acid (43). Treatment with the latter results in the formation of graphitic oxide, mellitic acid (benzene hexacarboxylic acid), hydrocyanic acid, carbon dioxide and nitrous oxide, depending on the experimental conditions. In other words, the whole structure of the carbon is probably altered, and not only its surface properties as determined by the nature of the surface organic functional groups. Therefore, it is not suprising that carbon treated with nitric acid loses all gold activity. Furthermore, the reduction in the gold capacity observed on addition to the solution of reducing agents such as hydroquinone and hydrazine, which are neutral molecules, need not necessarily be associated with the reducing power of these molecules per se and its effect on the nature of the surface functional groups. This loss of capacity could simply be due to a competition between gold cyanide and these neutral molecules for adsorption on the carbon, owing to the high affinity of carbon for the latter. In fact, modification of the commonly used eluant solutions for gold cyanide on carbon by addition to them (for example, 5 to 20 per cent of ethanol (11) or acetone to an aqueous cyanide-hydroxide solution) has a most beneficial effect on the rate and efficiency of the elution process.

Grabovskii et al. (44) found that gold cyanide adsorbed onto activated carbon prepared from a phenol-formaldehyde polymer could be effectively desorbed only if ligands such as $\mathrm{CN}^{-}$and $\mathrm{SCN}^{-}$, which are capable of forming strong complexes with gold, were present in the eluant. On the basis of this meagre evidence, they proposed that adsorption occurred by complete reduction of the gold cyanide complex to metallic gold, according to:

$$
\begin{aligned}
\mathrm{NaAu}(\mathrm{CN})_{2}+\mathrm{C}_{\mathrm{x}}+2 \mathrm{NaOH} \rightleftharpoons \\
\quad \mathrm{C}_{\mathrm{x}} \mathrm{O}--\mathrm{Na}+\mathrm{Au}+2 \mathrm{NaCN}+\mathrm{H}_{2} \mathrm{O}(12)
\end{aligned}
$$

where $\mathrm{C}_{\mathrm{x}} \mathrm{O}$--- $\mathrm{Na}$ represents the oxidized surface of the carbon with an adsorbed cation. This in itself is most improbable, because it is more likely that after supplying electrons for the reduction the carbon would become positively charged, thereby attracting an anion rather than a cation for charge balance. Nevertheless, the reducing power of the carbon was attributed to the presence of delocalized $\pi$-electrons in the aromatic graphite-like layers of the crystallites that form part of the proposed structure of activated 
carbon (45). However, the exact origin of the reducing power of certain activated carbons is, as yet, not fully understood.

Recently, Cho et al. (46 to 49) published the results of a detailed investigation of the kinetics of adsorption of $\mathrm{Au}(\mathrm{CN})_{2}^{-}$and $\mathrm{Ag}(\mathrm{CN})_{2}^{-}$on activated coconut shell carbon, and of the factors that influence the adsorption of $\mathrm{Ag}(\mathrm{CN})_{2}^{-}$(and by analogy $\mathrm{Au}(\mathrm{CN})_{2}^{-}$) on the same product. The adsorption of $\mathrm{Ag}(\mathrm{CN})_{2}^{-}$was found to be reversible, and therefore the adsorption reaction was considered to be subject more to physisorption than to chemisorption forces. Although these same authors had previously suggested that $\mathrm{Au}(\mathrm{CN})_{2}^{-}$was loaded onto carbon by an anion-exchange mechanism (38), in the light of the results of new experiments,

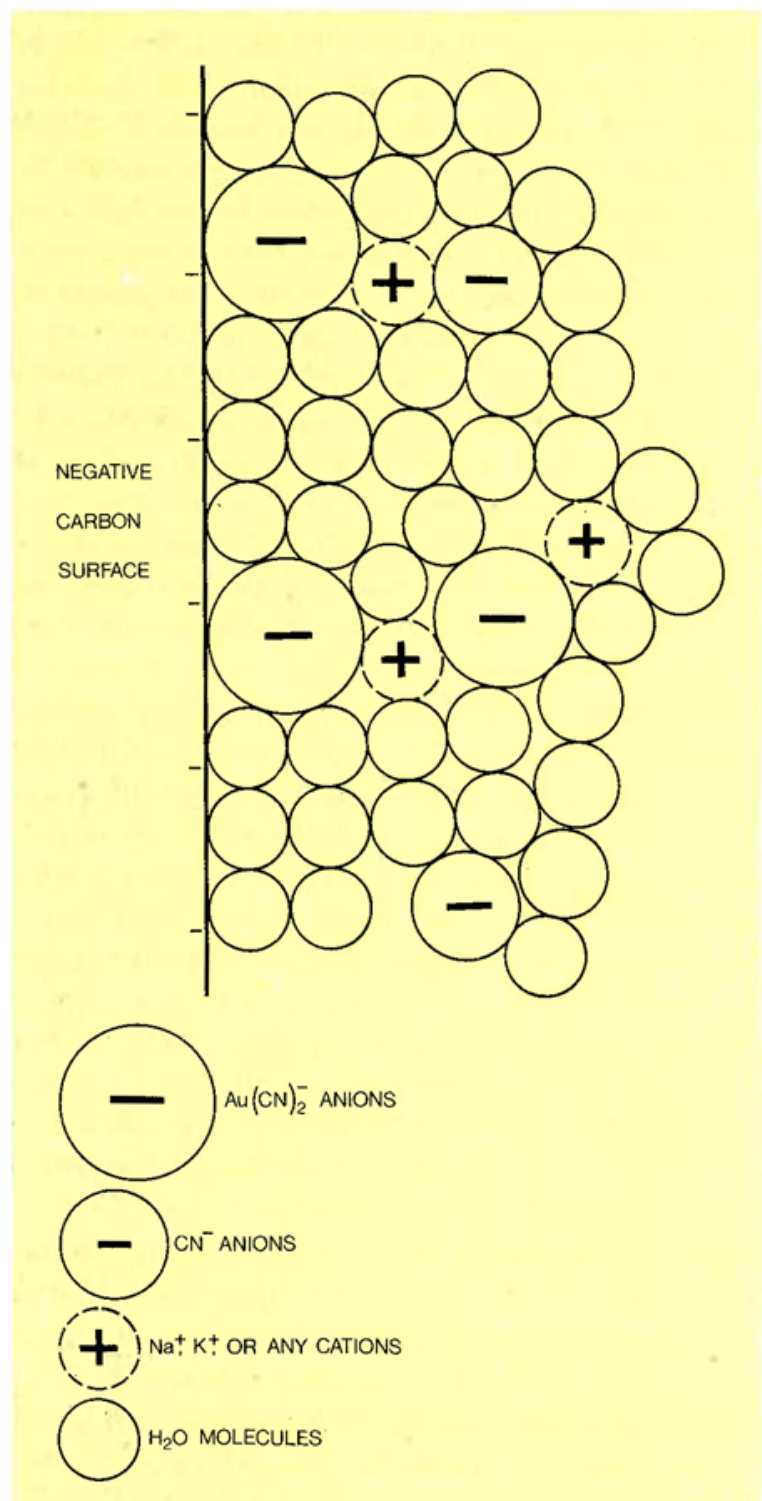

Fig. 6 Schematic representation of the model proposed by Cho (48) for the adsorption of $\mathrm{Au}(\mathrm{CN})_{2}^{-}$ anions onto activated coconut shell carbon they modified their earlier theories and now advanced a mechanism for the adsorption of both $\mathrm{Au}(\mathrm{CN})_{2}^{-}$and $\mathrm{Ag}(\mathrm{CN})_{2}^{-}$on carbon, which was in agreement with the ionic solvation-energy theory developed by Anderson and Bockris (50) to account for the specific adsorption of anions on metal electrodes.

According to this theory, the degree and type of ionic hydration are the principal factors that determine specific adsorption. Applied to carbon, the theory predicts that a large, weakly hydrated anion such as $\mathrm{Au}(\mathrm{CN})_{2}^{-}$will be specifically adsorbed on the carbon surface after losing some of its primaryhydration water molecules, whereas small anions with a large number of strongly bound water molecules in their primary-hydration shells, for example $\mathrm{CN}^{-}$, will not be specifically adsorbed and will therefore remain in the outer part of the electrical double layer. The capacity of carbon for $\mathrm{Ag}(\mathrm{CN})_{2}^{-}$was indeed observed to be considerably higher than for $\mathrm{CN}^{-}$, and Cho and Pitt (49) regarded this as proof of the applicability of their model. They also found that the small, highly hydrated $\mathrm{Na}^{+}$cation was adsorbed on the carbon only if it was present in solution with $\mathrm{Ag}(\mathrm{CN})_{2}^{-}$anions, and not if it was present with small, highly hydrated anions such as $\mathrm{CN}^{-}$. They obtained similar results with $\mathrm{Ca}^{2+}$ cations.

Confirming the results of other researchers $(24,37)$ on the adsorption of gold cyanide, Cho and Pitt (49) found that an increase in the concentration of $\mathrm{K}^{+}$, $\mathrm{Na}^{+}$and $\mathrm{Ca}^{2+}$ ions in solution enhanced the adsorption of silver cyanide on carbon. To account for these 'ionic strength' effects, they proposed that the silver cyanide complex was specifically adsorbed on the carbon surface and the cations in turn were nonspecifically adsorbed in the electrical double layer, and that these cations provided additional sites for the adsorption of anions such as $\mathrm{Ag}(\mathrm{CN})_{2}^{-}$(or $\mathrm{Au}(\mathrm{CN})_{2}^{-}$) and $\mathrm{CN}^{-}$. Thus, the new adsorption mechanism involved a multilayer formation of ions $(48,49)$ as depicted in Figure 6. The deleterious effect of free $\mathrm{CN}^{-}$anions on the adsorption of $\mathrm{Ag}(\mathrm{CN})_{2}^{-}$on carbon (although $\mathrm{CN}^{-}$is not supposed to be specifically adsorbed) was explained in terms of competition between $\mathrm{Ag}(\mathrm{CN})_{2}^{-}$and $\mathrm{CN}^{-}$anions for non-specific adsorption on the cation sites.

Cho and Pitt $(48,49)$ observed that the capacity of carbon for gold cyanide was about three times higher than for silver cyanide, and this was attributed to the larger ionic radius of gold $(137 \mathrm{pm})$ compared with that of silver $(126 \mathrm{pm})$, which is consistent with the solvation-energy theory. Thus, the decreasing order of strength of adsorption was found to be $\mathrm{Au}(\mathrm{CN})_{2}^{-}>\mathrm{Ag}(\mathrm{CN})_{2}^{-}>\mathrm{CN}^{-}$, which is the order of decreasing size of the anions. However, Cho and Pitt's theory ignores the fact that the presence of large concentrations of anions in solution, $1.5 \mathrm{M} \mathrm{Cl}^{-}$for 
example, does not depress the gold capacity of carbon, which, as pointed out by Kuzminykh and Tjurin (35), is inconsistent with the loading of the simple anionic complexes by electrostatic interactions. The ability of the $\mathrm{CN}^{-}$ion to depress the loading of $\mathrm{Ag}(\mathrm{CN})_{2}^{-}$on carbon noted by the former authors could just as well be explained through the formation of the more highly charged $\mathrm{Ag}(\mathrm{CN})_{3}^{2-}$ anion which would be more weakly loaded.

Zeta potential measurements (measurements of the effective charges on the surfaces of the carbons) on coconut shell carbon showed that as the $\mathrm{pH}$ was lowered, the zeta potential became more positive. The enhanced loading of $\mathrm{Ag}(\mathrm{CN})_{2}^{-}$and $\mathrm{Au}(\mathrm{CN})_{2}^{-}$ which was observed at acidic $\mathrm{pH}$ values was therefore attributed to electrostatic interactions between the gold and silver cyanide anions and the additional positive sites that presumably formed on the carbon surface owing to the irreversible adsorption of $\mathrm{H}^{+}$ ions. For example, Garten and Weiss (34) proposed that chromene groups on the surface of the carbon were converted to carbonium ions in the presence of acid and oxygen, in accordance with:

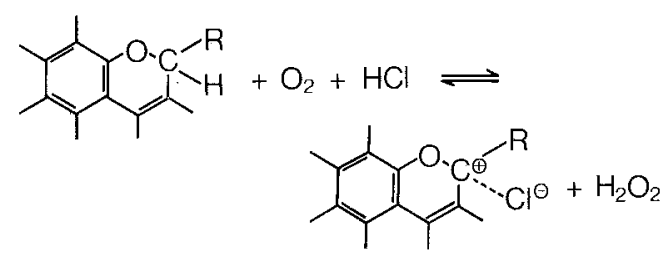

The object of the investigation was not to prove or disprove the validity of equation (13). It simply served as an explanation for the creation of positive sites on the carbon in the presence of acid, which would account for the observed rise in zeta potentials and enhanced gold loading.

The latest paper to be published in the field is that of McDougall et al. (24) who confirmed the enhancing effect that the addition of salts, even $\mathrm{KCN}$, and acids has on the gold capacity of activated coconut shell carbon compared with its capacity for gold from solutions containing only $\mathrm{KAu}(\mathrm{CN})_{2}$, as found by other research teams $(37,49)$. However, they demonstrated that large, weakly hydrated anions similar to $\mathrm{Au}(\mathrm{CN})_{2}^{-}$, for example $\mathrm{ClO}_{4}^{-}$, present in the adsorption medium even in large concentrations, did not depress the gold capacity of the carbon, whereas the gold capacity of a typical strong base anionexchange resin, for example IRA-400 (with quaternary ammonium functional groups), was severely affected. McDougall et al. therefore concluded that gold adsorption on coconut shell carbon could not be attributed to simple electrostatic interactions between $\mathrm{Au}(\mathrm{CN})_{2}^{-}$anions and positively charged sites on the surface of the carbon as proposed by numerous workers $(34,35,39,48,49)$. This conclusion was also supported by their finding that, contrary to normal anion-exchange behaviour, the capacity of carbon for the $\mathrm{Au}(\mathrm{CN})_{2}^{-}$anion was sensitive to temperature (37, 38), the adsorption reaction being exothermic to the extent of $42 \mathrm{~kJ} / \mathrm{mol}$ for gold concentrations in the range 50 to $800 \mathrm{mg} / \mathrm{l}$. They pointed out that the exothermic nature of the adsorption process also explained why gold cyanide was effectively eluted only at elevated temperature. However, the fact that $\mathrm{KAu}(\mathrm{CN})_{2}$ is fourteen times more soluble in hot water than in cold water could equally well account for this observation.

By applying the technique of X-ray photoelectron spectroscopy (XPS) to gold-loaded carbons, McDougall et al. were able to ascertain that the oxidation state of the gold in the gold cyanide adsorbate was neither I as required for the presence of $\mathrm{Au}(\mathrm{CN})_{2}$ or $\mathrm{AuCN}$ on the carbon, nor 0 which would indicate the presence of metallic gold on the carbon, but an intermediate 0.3 , irrespective of loading conditions. On the basis of this result, the authors proposed that reduction, albeit partial reduction, was an important aspect of the loading mechanism.

Further credibility was given to this proposal when the same authors found a correlation between the gold capacities of a series of commercially available activated carbon products that were prepared from a variety of raw materials and their reduction potentials as measured by a graphite rod technique. However, in order to account for the observed cation and acid effects, namely the ionic-strength effects, they postulate that the adsorption mechanism is a two-step process. The initial stage of this involves the adsorption of an ion-pair $\mathrm{M}^{\mathrm{n}+}\left[\mathrm{Au}(\mathrm{CN})_{2}^{-}\right]_{\mathrm{n}}$ onto the carbon. The effect of the nature of $M^{n+}$ on the adsorption is related to solubility considerations, the carbon preferring species with low solubilities. If $\mathrm{M}^{\mathrm{n}+}$ is $\mathrm{Ca}^{2+}$ or $\mathrm{H}^{+}$, a product with a lower aqueous solubility is obtained than if $\mathrm{M}^{\mathrm{n}+}$ is $\mathrm{Na}^{+}$or $\mathrm{K}^{+}$and this accounts for the observed trends. Once the gold cyanide complex is in the carbon, McDougall et al. visualize it as being reduced in a second step to some unidentified species. However, they postulate that this may be either a substoichiometric AuCN polymer with metallic gold in the matrix or a cluster compound of gold containing gold atoms in both the 0 and I formal oxidation states. Such compounds with triphenylphosphine $\mathrm{P}(\mathrm{Ph})_{3}$ are well known for gold, for example $\mathrm{Au}_{11}(\mathrm{CN})_{3}\left[\mathrm{P}(\mathrm{Ph})_{3}\right]_{7}$, and their XPS spectra somewhat resemble those for the gold-loaded carbons. The presence on the carbon of a gold cyanide complex which has lost cyanide also accounts for observations $(24,44)$ that complete desorption of gold from carbon requires the presence of an agent such as $\mathrm{CN}^{-}$or $\mathrm{SCN}^{-}$in the eluant.

An interesting finding was that in the adsorption on carbon of neutral inorganic molecules such as 
$\mathrm{Hg}(\mathrm{CN})_{2}$, the addition of salts or acids to the adsorption medium does not affect the loading capacity of the carbon for the mercury complex, whereas marked ionic strength and acidic effects are characteristic of the loading behaviour of anions such as $\mathrm{Au}(\mathrm{CN})_{2}^{-}$on carbon. Furthermore, the neutral mercury complex was found to compete directly with $\mathrm{Au}(\mathrm{CN})_{2}^{-}$anions for adsorption sites when they were present together in solution, which led McDougall et al. to suggest that the negative charge of the gold cyanide anion was not essential for adsorption onto activated carbon.

\section{Concluding Remarks}

The reader of this review will quite correctly have gained the impression that there is much confusion and little agreement between the various researchers in the field concerning the details of the mechanism by which activated carbon loads gold cyanide. However, amidst the confusion it can at present be reasonably concluded that those theories in which the complete reduction of the $\mathrm{Au}(\mathrm{CN})_{2}^{-}$complex to metallic gold on the carbon is postulated can be discounted, primarily because the most negative measured value of the potential of thermally activated carbon products $(-0.14 \mathrm{~V}$ against SCE) is considerably more positive than the standard reduction potential for $\mathrm{Au}(\mathrm{CN})_{2}^{-}(-0.85 \mathrm{~V}$ against SCE). Other evidence in support of this conclusion is that the chemicals, such as sodium sulphide, which have been found to be good eluants for gold cyanide on carbon do not desorb metallic gold from carbon. Moreover, a gold cyanide-loaded carbon has been observed to turn a golden colour on heating only (32), which is reminiscent of the behaviour of $\mathrm{KAu}(\mathrm{CN})_{2}$ and $\mathrm{AuCN}$.

On the other hand, the observation that the presence in the gold cyanide solution of large concentrations of anions, such as $\mathrm{Cl}^{-}$or $\mathrm{ClO}_{4}^{-}$, does not depress the loading of $\mathrm{Au}(\mathrm{CN})_{2}^{-}$on carbon despite the fact that from electrostatic considerations they should be competitive (especially the $\mathrm{ClO}_{4}^{-}$anion which like $\mathrm{Au}(\mathrm{CN})_{2}^{-}$is large and weakly hydrated), militates against those mechanisms which propose loading as a result of electrostatic interactions between the negatively charged gold cyanide anions and positively charged sites on the surface of the carbon (by whatever mechanism such positively charged sites may arise on the carbon surface).

Other mechanisms are all possible, individually or in combination. They include (a) loading of $\mathrm{M}^{\mathrm{n}+}\left[\mathrm{Au}(\mathrm{CN})_{2}\right]_{\mathrm{n}}$ as an ion-pair or as a neutral molecule, by either adsorption on the large internal surface of the carbon or precipitation of the complex in the pores of the carbon, with $\mathrm{M}^{\mathrm{n}+}$ derived from the solution or from the ash content of the carbon; (b) chemical degradation of the $\mathrm{Au}(\mathrm{CN})_{2}^{-}$anions to insoluble AuCN which is retained in the pores of the carbon (the involvement of carbon monoxide as proposed in the early theories is unlikely); and (c) partial reduction of the gold cyanide complex to some compound containing gold atoms in both the 0 and I formal oxidation states.

The confusion which exists in the literature can to a large extent be ascribed to the fact that activated carbon is not readily amenable to direct investigation by such techniques as infra-red spectroscopy or X-ray diffraction, so that very little is known about the adsorbent itself. For instance, not even the identities of the oxygen-containing organic functional groups that are formed on the carbon during activation are known with certainty, let alone the exact nature of the gold cyanide adsorbate. A further complication is the fact that investigations have been conducted on carbon products prepared from a range of raw materials with ash contents differing in composition and quantity, and activated under a variety of conditions. It is therefore possible that different mechanisms are responsible for gold adsorption, depending on the nature of the activated carbon itself. Furthermore, it is also possible that the mechanism of adsorption is affected by the initial concentration of gold in solution. As a result, in the past, researchers have been free to speculate on how the bewildering array of experimental evidence might be explained. Any future meaningful advances will almost certainly come about only as a result of the successful application of more direct techniques to well-characterized and uniform carbon products, which have been loaded with gold cyanide under well-defined and reproducible experimental conditions.

\section{References}

W. N. Davis, U.S. Patent 227, 963 (1880)

2 'Gold Metallurgy in South Africa', edited by R. J. Adamson, Chamber of Mines of South Africa, Johannesburg, 1972, p. 88

3 W D. Johnson, U.S. Patent 522, 260 (1894)

4 A. W. Allen, Metall. Chem. Eng., 1918, 18, (12), 462-644

5 G. J. McDougall and R. D. Hancock, Miner. Sci. Eng., 1980 $12,(2), 85-99$

6 J. B. Zadra, 'A Process for the Recovery of Gold from Activated Carbon by Leaching and Electrolysis', U.S. Bureau of Mines, Washington, D.C., R.I. No. 4672, 1950

7 J. B. Zadra, A. K. Engel and H. J. Heinen, 'A Process for Recovering Gold and Silver from Activated Carbon by Leaching and Electrolysis', U.S. Bureau of Mines, Washington, D.C., R.I. No. 4843, 1952

8 K. B Hall, World Min., 1974, 27, (12), 44-49

9 P. A. Laxen, G. S. M. Becker and R. Rubin, F. S. Afr. Inst Min. Metall., 1979, 79, (11), 315-326

10 R. J. Davidson and D. Duncanson, 7. S. Afr. Inst. Min Metall., 1977, 77, (12), 254-261

11 H. J. Heinen, D. G. Peterson and R. E. Lindstrom, in 'World Mining and Metals Technology, Vol. 1', edited by A. Weiss, AIME, New York, 1976, pp. 551-564

12 J. R. Ross, H. B. Salisbury and G. M. Potter, 'Pressure Stripping Gold from Activated Carbon', presented at the Society of Mining Engineers, AIME Annual Conference, Chicago, IL., 1973, February

13 A. J. Gilmore, Can. Min. F., 1969, 90, (6), 78-80 
14 D. I. Nicol, f. S. Afr. Inst. Min. Metall., 1979, 79, (17), 497.502

15 T. G. Chapman, U.S. Patent 2,147,009 (1939)

16 See (2) p. 125

17 H. J. Heinen, D. G. Peterson and R. E. Lindstrom, U.S Bureau of Mines, Washington, D.C., I.C. No. 8770, 1978

18 D. W. Kappes and R. W. Eveleth, Min. Eng., 1979, 31, (2), 136-140

19 J. S. Mattson and H. B. Mark, 'Activated Carbon', Marcel Dekker, New York, 1971

20 J. C. Bokros, in 'Chemistry and Physics of Carbon, Vol. 5', edited by P. C. Walker, Marcel Dekker, New York, 1969, pp. $1-118$

21 H. P. Boehm, Adv. Catal., 1966, 16, 179-274

22 H. P. Boehm, E. Diehl, W. Heck and R. Sappok, Angez. Chem., Int. Ed., 1964, 3, 669-678

23 V. A. Garten, D. E. Weiss and J. B. A. Willis, f ust. 7. Chem., 1957, 10, 295-308

24 G. J. McDougall, R. D. Hancock, M. J. Nicol, O. L. Wellington and R. G. Copperthwaite, f. S. Afr. Inst. Min. Metall., 1980, 80, (9), 344-356

25 S. Brussov, Z. Chem. Ind. Kolloide, 1909, 5, 137-141

26 M. Green, Trans. Inst. Min. Metall., 1913-1914, 23, 65-84

27 H. R. Edmands, Trans. Inst. Min. Metall., 1917-1918, 27, 277-325

28 W. R. Feldtmann, Trans. Inst. Min. Metall., 1914-1915, 24, 329-358

29 C. R. A. Clauss and K. Weiss, 'Adsorption of Aurocyanide on Carbon', Council for Scientific and Industrial Research Pretoria, Report of Investigation No. CENG. 206, 1977, September

30 L. B. Williams, Min. Mag., London, 1923, 23, 139-147

31 E. G. R. Ardagh, f. Soc. Chem. Ind., 1921, 15, (October)

32 J. Gross and J. W. Scott, U.S. Bureau of Mines, Washington, D.C., Technical Paper No. 378, 1927
33 J. E. Huff, E. G. Fochtman and J. M. Bigger, in "Carbon Adsorption Handbook', edited by P. N. Cheremisinoff, Ann Arbor Science Publishers Inc., 1978

34 V. A. Garten and D. E. Weiss, Rev. Pure Appl. Chem., 1957, 7, (June), 69-121

35 V. M. Kuzminykh and N. G. Tjurin, Izv. Vyssh. Ucheb. Zaved., Tsvet. Metall., 1968, 11, (4), 65-70

36 V. M. Kuzminykh, N. G. Tjurin and A. A. Nikulova, f. Appl. Chem. USSR, 1969, 42, (9), 1886-1888

37 R. J. Davidson, J. S. Afr. Inst. Min. Metall., 1974, 75, 67-76

38 S. Dixon, E. J. Cho and C. H. Pitt, 'The Interaction between Gold Cyanide and High Surface Area Charcoal', AIChE Meeting, Chicago, IL., 1976, November 26-December 2

40 A. Frumkin, Kolloid Z., 1930, 51, 123-129

41 A. Frumkin, B. Burshtein and P. Lewin, Z. Phys. Chem., 1931, 157A, 422-427

42 B. Burshtein and A. Frumkin, Z. Phys. Chem., 1929, 141A, 219-225

43 C. L. Mantell, 'Carbon and Graphite Handbook', Interscience Publishers, John Wiley \& Sons Inc., 1968

44 A. I. Grabovskii, S. L. Grabchak, L. S. Ivanova, R. K. Storozhuk and V. M. Shirshov, Zh. Prikl. Khim., $1977, \mathbf{5 0},(3), 522-524$

45 A. I. Grabovskii and L. S. Ivanova, Zh. Prikl. Khim., 1978, 51, (7), 1515-1518

46 E. Cho, S. N. Dixon and C. H. Pitt, Metall. Trans., 1979, 10B, 185-189

47 E. Cho and C. H. Pitt, Metall. Trans., 1979, 10B, 165-169

48 E. Cho, 'Adsorption of Silver Cyanide on Activated Carbon', Ph.D. Thesis, University of Utah, 1978

49 E. Cho and C. H. Pitt, Metall. Trans., 1979, 10B, 159-164

50 T. N. Anderson and J. O'M. Bockris, Electrochim. Acta, $1964,9,(4), 347-354$

51 R. J. Davidson, V. Veronese and M. V. Nkosi, J. S. Afr. Inst. Min. Metall., 1979, 79, (10), 281-297 